\title{
Instantons, the QCD Vacuum, and Hadronic Physics*
}

\author{
J. W. Negele ${ }^{\text {a }}$ \\ ${ }^{a}$ Center for Theoretical Physics, Laboratory for Nuclear Science, and Department of Physics, \\ Massachusetts Institute of Technology, 77 Massachusetts Ave., Cambridge, MA 02139, USA
}

A large body of evidence from lattice calculations indicates that instantons play a major role in the physics of light hadrons. This evidence is summarized, and recent results concerning the instanton content of the $\mathrm{SU}(3)$ vacuum, instanton contributions to the static potential, and a new class of instanton solutions at finite temperature are reviewed.

\section{INTRODUCTION AND PHYSICAL PICTURE}

\subsection{Physics motivation}

One of the great advantages of the path integral formulation of quantum mechanics and field theory is the possibility of identifying nonperturbatively the stationary configurations that dominate the action and thereby identify and understand the essential physics of complex systems with many degrees of freedom. Thus, the discovery of instantons in 1975 [1] gave rise to great excitement and optimism that they were the key to understanding QCD. Indeed, in contrast to other many body systems in which the quanta exchanged between interacting fermions can be subsumed into a potential, it appeared that QCD was fundamentally different, with topological excitations of the gluon field dominating the physics and being responsible for a host of novel and important effects including the $\theta$ vacuum, the axial anomaly, fermion zero modes, the mass of the $\eta^{\prime}$, and the chiral condensate. However, despite nearly a quarter of a century of theoretical effort, it has not been possible to proceed analytically beyond the dilute instanton gas approximation [2]. In the intervening years, the instanton liquid model [3 [5] provided a successful phenomenology and qualitative physical understanding, but a quantitative exploration of the role of instantons in nonperturbative QCD has had to

\footnotetext{
*This work is supported in part by the U. S. Department of Energy (DOE) under cooperative research agreement \#DE-FC02-94ER40818.
}

wait until lattice QCD became sufficiently sophisticated and sufficient resources could be devoted to the study of instanton physics.

In recent years, significant progress has been made by a number of groups in understanding instanton physics on the lattice, and this review summarizes some of the highlights. This field is both exciting and frustrating. It is exciting because, at last, a substantial body of evidence shows that the original vision of the role of instantons in QCD is borne out in nature. However, as will be clear below, it is also frustrating because of intrinsic limits to the precision with which one can apply ultimately semiclassical concepts. Since there is more interesting material than could be accommodated, I will omit significant developments in spectral methods, which are covered in another talk [6], and recent work on abelian projection and monopoles, a subject to which I am not able to provide any additional insight.

\subsection{Aspects of continuum instanton physics}

To put lattice investigations in context, it is useful to recall relevant aspects of continuum instanton physics. Working in Euclidean time, we evaluate a path integral of the form $\int \mathcal{D}[A] e^{\int d^{4} x S[A]}$. Hence, as in statistical mechanics, the weight of a configuration depends not only on its energy, but also on its entropy - the number of ways it can be realized. In addition, tunneling solutions arise as periodic classical solutions in an inverted potential. Vortices in two- 
dimensional spin systems provide a useful analogy. Classified by winding number, the cost of the localized core energy is compensated by entropy so that vortices can become the dominate degrees of freedom as in the Kosterlitz-Thouless phase transition. Similarly, we may expect BPST instantons [1] which connect degenerate minima of differing winding number with the self-dual gauge potential $A_{\mu}^{a}(x)=\frac{2 \eta_{a \mu \nu} x_{\nu}}{x^{2}+\rho^{2}}$ having scale-invariant action $S=\frac{1}{4} \int d^{4} x F_{\mu \nu}^{a} F_{\mu \nu}^{a}=8 \pi^{2} g^{-2} \equiv S_{0}$ to have a significant presence in the vacuum due to the high entropy associated with translation, size, and color orientation. The action and topological charge density are localized around the center as $\pm F_{\mu \nu}^{a} \tilde{F}_{\mu \nu}^{a}=F_{\mu \nu}^{a} F_{\mu \nu}^{a}=\frac{192 \rho^{4}}{\left(x^{2}+\rho^{2}\right)^{4}}$. The tunneling rate is $d n_{I} \sim\left(\frac{8 \pi^{2}}{g^{2}}\right)^{2 N_{c}} \frac{d \rho}{\rho^{5}} d^{4} x^{(I)} e^{-\frac{8 \pi^{2}}{g^{2}\left(\Lambda^{-1}\right)}}(\Lambda \rho)^{\frac{11 N_{c}}{3}}$, where the prefactor and the running of the coupling constant in the last factor produce a distribution of instantons $\sim \rho^{6}$ for $\mathrm{SU}(3)$. Physically, we expect this distribution to be cut off at large $\rho$ by interactions between instantons and by fluctuations when the amplitude of a sufficiently large instanton becomes small relative to quantum fluctuations. It is the difficulty in treating these infrared effects that has stymied analytic progress.

From the axial anomaly, $\partial_{\mu} \sum_{f} \bar{\psi} \gamma_{\mu} \gamma_{5} \psi=2 m \sum_{f} \bar{\psi} \gamma_{5} \psi+\frac{N_{f}}{16 \pi^{2}} F_{\mu \nu}^{a} \tilde{F}_{\mu \nu}^{a}$, the topological charge satisfies the index theorem and, for periodic systems, may be expressed in terms of fermion eigenfunctions

$Q=\frac{g^{2}}{32 \pi^{2}} \int F \tilde{F}=n_{L}-n_{R}=m \sum_{\lambda} \frac{\int \psi_{\lambda}^{\dagger}(x) \gamma_{5} \psi_{\lambda}(x)}{m+i \lambda}$, where $n_{L}$ and $n_{R}$ denote the number of fermion zero modes. For an isolated instanton, the zero mode is $\psi_{0}(x)=\frac{\rho \gamma \cdot \hat{x}\left(1+\gamma_{5}\right)}{2 \pi\left(x^{2}+\rho^{2}\right)^{3 / 2}} \phi$. In the limit of light quarks, the Greens function for $\mathrm{N}_{f}$ quarks reduces to the product of zero modes $\prod_{f} \operatorname{Det}[\not D+m] \bar{\psi}_{f}(x) \psi_{f}(y) \underset{m \rightarrow 0}{\longrightarrow} \prod_{f} \psi_{0}(x) \psi_{0}^{\dagger}(y)$ and gives rise to the 't Hooft interaction. Thus, light quarks propagate by zero modes which in turn arise from instantons. Based on large $\mathrm{N}$ arguments, the Veneziano-Witten formula [7, 8] relates the $\eta^{\prime}$ mass to the topological susceptibility in the pure gluon sector

$\chi \equiv \int \frac{d^{4} x}{V}\langle Q(x) Q(0)\rangle=\frac{f_{\pi}^{2}}{2 N_{f}}\left(m_{\eta}^{2}+m_{\eta^{\prime}}^{2}-2 m_{K}^{2}\right)$ yielding the expectation that $\chi=(180 \mathrm{MeV})^{4}$.
Finally, for an ensemble of instantons and antiinstantons, we expect mixing of their zero modes to generate a finite density of states near zero virtuality. Hence, by the Banks-Casher relation 9], $-\langle\bar{\psi} \psi\rangle=\int d \lambda \rho(\lambda) \frac{2 m}{\lambda^{2}+m^{2}} \underset{m \rightarrow 0}{\longrightarrow} \pi \rho(\lambda=0)$, they account for the chiral condensate. Subsequent sections will discuss the extent to which this continuum instanton physics can now be seen on the lattice.

\subsection{The instanton liquid}

The instanton liquid model [3 5 ] provides an economical phenomenology of instanton mediated quark propagation in the QCD vacuum. The integral over all gluon fields that one evaluates in lattice QCD using an ensemble of configurations sampling the action is replaced by an ensemble of instanton and anti-instanton configurations. Although the model has been refined to account for interactions between instantons and the fermion determinant, the gross features can be seen for the case of an ensemble of instantons and anti-instantons of size $\rho \sim 1 / 3 \mathrm{fm}$ and density $n \sim 1 \mathrm{fm}^{4}$ randomly distributed in space and color orientation, where the values of $\rho$ and $n$ are determined from the physical gluon and chiral condensates.

Due to the opposite shifts of left and righthanded fermion levels in the presence of an instanton, one may think of the 't Hooft interaction as a vertex that absorbs left-handed particles of each flavor and creates corresponding right-handed particles, and vice versa for antiinstantons. Mesons then propagate in the QCD vacuum by the hopping of quark-antiquark pairs between these vertices, and the qualitative features of the channel dependence arises naturally. Considering two flavors for simplicity, a spin-zero pion propagates by a $u_{R} \bar{d}_{L}$ pair interacting with an anti-instanton to produce a $u_{L} \bar{d}_{R}$ pair which in turn interacts with an instanton to return to a $u_{R} \bar{d}_{L}$ pair. Since the 't Hooft vertex is most attractive in this channel and the interaction can act in all orders, the pion is the most strongly attractive meson channel. In contrast, the spinone rho propagates only in second order, with an anti-instanton taking a $u_{R} \bar{d}_{R}$ pair to a $u_{L} d_{L} \bar{d}_{R} \bar{d}_{R}$ state which is then returned to a $u_{R} \bar{d}_{R}$ pair by 
an instanton, so we expect the interaction in this channel to be much weaker. Similarly, the scalar meson channel is repulsive and one expects the nucleon channel to be more attractive than the delta channel. The chiral condensate arises naturally in this picture by the fact that the zero modes for isolated instantons mix in the instanton liquid giving rise to a finite density of states at low virtuality.

The tendency of instanton-anti-instanton pairs connected by light quarks and antiquarks to form dipoles oriented in the thermal direction suggests a possible mechanism for chiral symmetry restoration at high temperature and may be manifested by dipoles wrapped around the periodic thermal direction above $\mathrm{T}_{\mathrm{C}}$.

\section{EVIDENCE FOR INSTANTONS IN HADRON STRUCTURE}

The expectations raised by the our analytical understanding of the role of instantons in QCD and the physical picture of quarks hopping between zero modes associated with instantons have now been borne out by a broad range of lattice calculations as described below.

\subsection{Vacuum current correlation functions}

Vacuum correlation functions for space-like separated hadron currents calculated in lattice QCD display the qualitative behavior expected from the 't Hooft interaction and agree semiquantitatively with the instanton liquid model. As emphasized in ref 10], correlation functions of the form $R(x) \equiv\left\langle 0\left|T J_{\mu}(x) J_{\mu}(0)\right| 0\right\rangle$ characterize the spatial and channel dependence of the interaction between quarks and antiquarks and thus supplement hadron bound state properties like phase shifts supplement deuteron properties in characterizing the nuclear interaction. The ratio $R(x) / R_{0}(x)$ of the interacting to free correlator has been calculated in quenched $\mathrm{QCD}$ for the following meson and baryon currents [11], $J=\bar{u} \gamma_{\mu} d, \bar{u} \gamma_{\mu} \gamma_{5} d, \bar{u} \gamma_{5} d, \bar{u} d, \epsilon_{a b c}\left[c^{a} C \gamma_{\mu} u^{b}\right] \gamma_{\mu} \gamma_{5} d^{c}$, and $\epsilon_{a b c}\left[u^{a} C \gamma_{\mu} u^{b}\right] u^{c}$. Results are consistent with dispersion analysis of $\mathrm{e}^{+}-\mathrm{e}^{-}$and other data in relevant channels, and typical results are shown in Fig. 1.

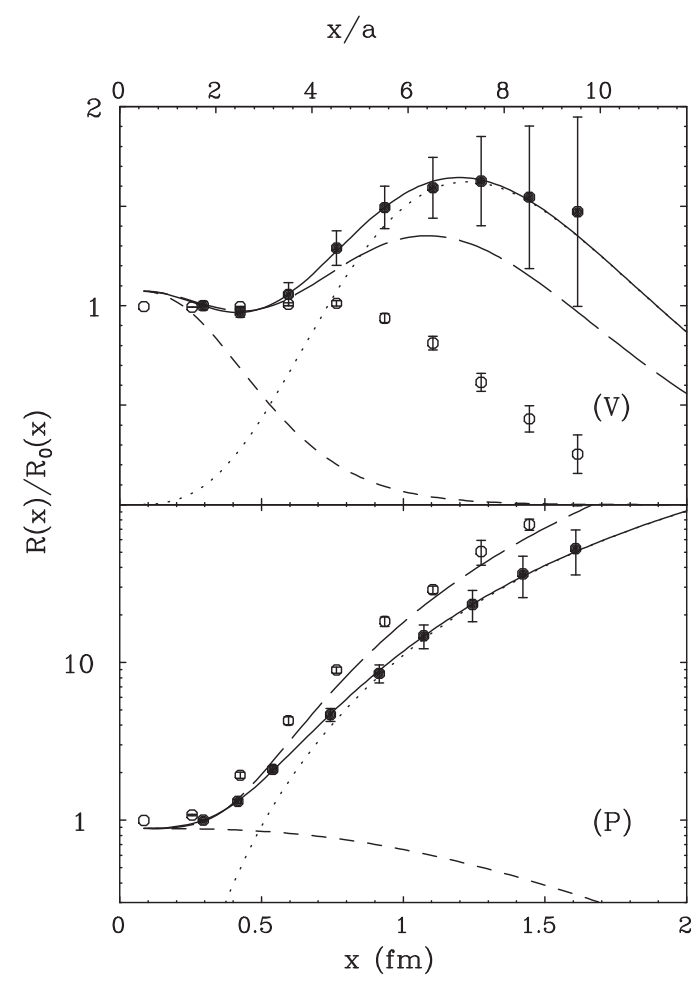

Figure 1. Vector $(V)$ and Pseudoscalar $(P)$ correlation functions from lattice calculations 11 (solid circles), from the random instanton model 12 13 (open circles) and from dispersion analysis 10 (long dashes). The solid curve fit to the lattice data is made up of a continuum contribution (short dashes) plus a resonance contribution (dotted curve).

Note for subsequent reference, that the solid curve fit to the lattice data may be decomposed into a continuum contribution concentrated near the origin (short dashed line) and a resonance contribution arising from the rho or pion which dominates in the region of $1 \mathrm{fm}$ and beyond (dotted line). Quenched calculations at $\beta=6.2$ 14 corroborate the original $\beta=5.7$ results.

\subsection{Comparison of results with all gluons and only instantons}

One dramatic indication of the role of instantons in light hadrons is to compare observables calculated using all gluon contributions 

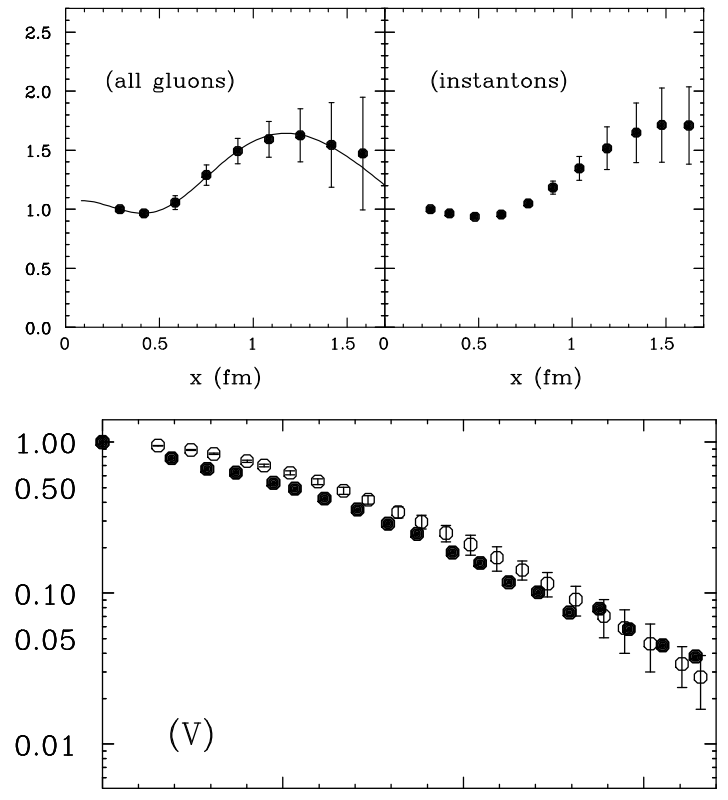

Figure 2. Vacuum correlator in the rho channel calculated with all gluons (upper left) and with only instantons (upper right) 17. The rho meson ground state density-density correlation function calculated with all gluons (solid circles) and with only instantons (open circles) is shown below.

with those obtained using only the instantons remaining after cooling. The basic idea of cooling [15, 16], and the variants described in Section 3 , is to iteratively locally relax a lattice gluon configuration and thereby approach the nearest local minimum of the classical action. Thus, one may extract the instanton content by removing local fluctuations while preserving topologically stable excitations. When a $\beta=5.7$ quenched configuration is cooled 25 steps, the gluon content changes dramatically [17]: the fluctuations in the action and topological charge densities decrease two orders of magnitude, self-dual instantons and antiinstantons become apparent, $\frac{\langle S\rangle}{S_{0}}$ decreases from over 20,00 to 64 , the string tension reduces to one fourth its original value, the lattice spacing defined by the nucleon mass decreases from $0.168 \mathrm{fm}$ to $0.142 \mathrm{fm}$, and the magnetic hyperfine components of the quark-quark potential become essentially zero. Hence, for example, the energies and wave functions of charmed and $B$ mesons would be drastically changed.

As shown in Fig. 2, however, the properties of the rho meson are virtually unchanged. The vacuum correlation function in the rho (vector) channel and the spatial distribution of the quarks in the rho ground state, given by the ground state density-density correlation function [18] $\left\langle\rho\left|\bar{q} \gamma_{0} q(x) \bar{q} \gamma_{0} q(0)\right| \rho\right\rangle$, are statistically indistinguishable before and after cooling. Also, as shown in Ref. 17, the rho mass is unchanged to within its $10 \%$ statistical error. In addition, the pseudoscalar, nucleon, and delta vacuum correlation functions and nucleon and pion densitydensity correlation functions are also qualitatively unchanged after cooling, except for the removal of the small Coulomb induced cusp at the origin of the pion. Similarly, the axial charge matrix elements specifying the spin content of the nucleon, $\left\langle\vec{P} \vec{S}\left|\left(\bar{q} \gamma^{i} i \gamma_{5} q\right)\right| \vec{P} \vec{S}\right\rangle=2 S^{i} \Delta q$, are quite similar when calculated with all gluons and only instantons [19]. This result suggests that when all the quark and gluon contributions are calculated, instantons, which are the natural mechanism to remove helicity from the valence quarks and transfer it to gluons and sea quarks, may account for the proton "spin crisis".

\subsection{Zero mode zone expansion}

Because, as discussed in Section 3, cooling somewhat modifies the instanton distribution, it is useful to study the zero modes of the original uncooled configuration directly. Several studies [6,20 22] calculate zero modes on the real axis and conjugate pairs displaced slightly off the axis due to the mixing of instantons and antiinstontons, examine the discrete lattice counterpart of the index theorem, and consider the presently unsolved problem of separating physical continuum solutions from doublers. A clear indication of the role of zero modes in light hadron observables is the degree to which truncation of the expansion $\sum_{\lambda} \frac{\psi_{\lambda}(x) \psi_{\lambda}(y)^{\dagger}}{m+i \lambda}$ to the zero mode zone reproduces the result using the complete propagator. The top plot in Fig. 3 shows the effect of including the lowest 16 through 128 of the 786,432 modes on a $16^{4}$ lattice in the vector correlation function, indicating that already 64 modes repro- 

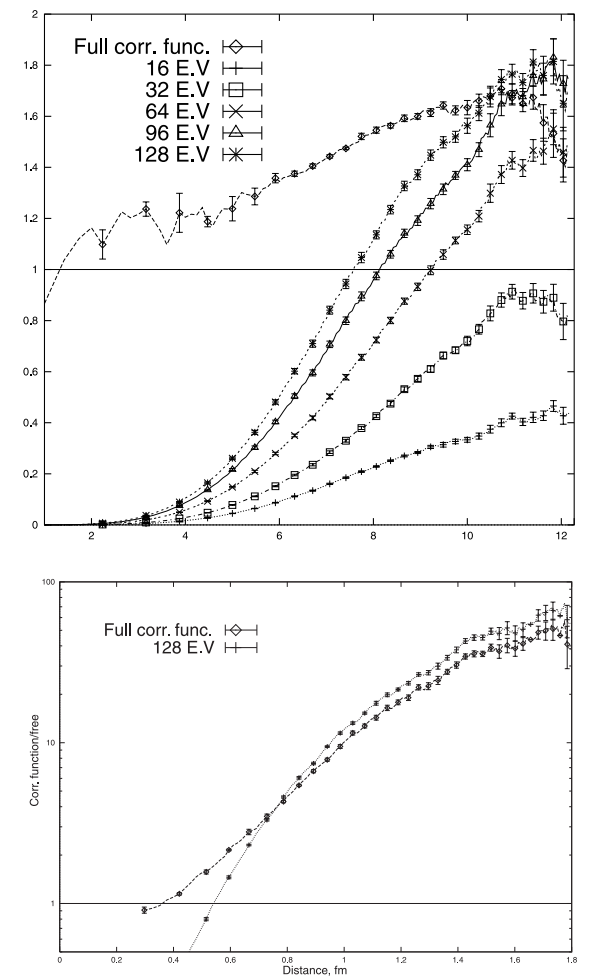

Figure 3. Truncated eigenmode expansion [20].

duce most of the strength of the rho peak and 128 modes produce the full strength. The lower plot shows that 128 modes also reproduce the full pion strength 20]. Similarly, most of the strength of the disconnected graph contribution to the $\eta^{\prime}$ correlation function, which should be particularly sensitive to instantons, is already provided by the lowest 32 eigenmodes, and the fermionic definition of the topological charge is nearly saturated by the lowest 8 modes 21 .

\subsection{Localization of zero modes}

The observation of localization of low eigenmodes of uncooled configurations at the locations of instantons identified by cooling confirms several important ideas. Even in the presence of fluctuations several orders of magnitude larger than the instanton fields themselves, the light quarks essentially average out these fluctuations and produce localized peaks at the topological excitations as expected from semiclassical arguments. In addition, it is clear that the instantons which sur-

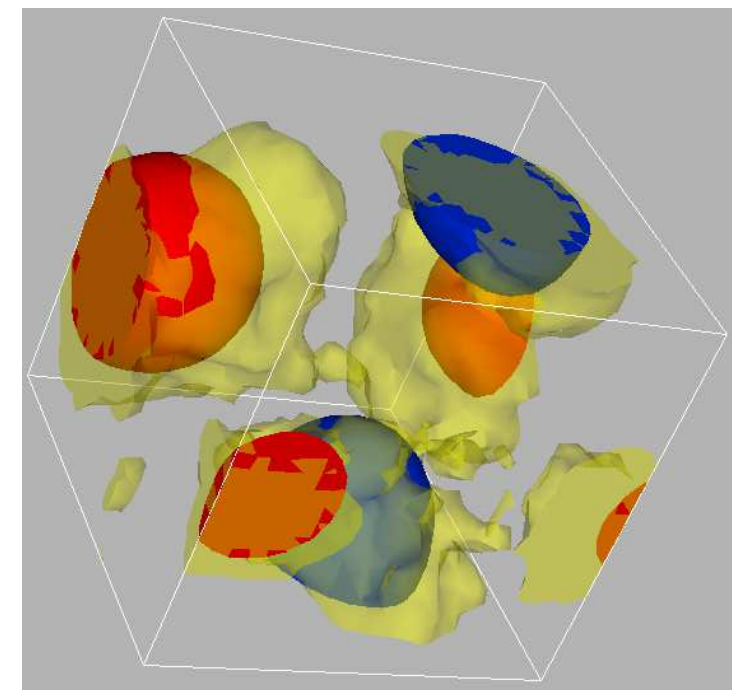

Figure 4. Isosurfaces on a periodic lattice of topological charge density $F \tilde{F}(x)$ calculated from a cooled configuration (dark surfaces) and $\bar{\psi}_{0}(x) \psi_{0}(x)$ calculated on the corresponding uncooled configuration (light surfaces) 23.

vive cooling represent topological structure in the original configuration. This localization has been observed for unquenched Wilson fermions at $\mathrm{T}$ $=0, \beta=5.5$ [20] and for unquenched staggered fermions at $\mathrm{t} \leq \mathrm{T}_{c}$ at $\beta=5.65$ [23. Isosurfaces of $\bar{\psi}_{0}(x) \psi_{0}(x)$ for the lowest uncooled eigenmode [23] are compared with the cooled topological charge density in Fig. 4 .

\subsection{Molecular correlations}

At high temperature, the fermion determinant favors alignment of instanton-anti-instanton pairs in the thermal direction 10]. Although aligned molecules have not been observed directly in the topological charge density of cooled configurations, a suggestive anisotropy in the topological susceptibility measured on differently oriented sublattices was reported at this conference [23]. Lattice slices that are narrow in the time direction but span the full spatial volume, which would have a large range in the value of $\mathrm{Q}$ for a dipole layer, were observed to have $\left\langle Q^{2}\right\rangle$ up to twice as large as slices that are narrow in one spatial direction but span the full time range, which 
would tend to have canceling positive and negative charges from the dipoles in the slice. Whereas it is not presently understood why a comparable asymmetry is also observed in quenched $\mathrm{SU}(2)$, subvolume susceptibility measurements are an interesting and promising development.

\subsection{Spectral density and $\langle\bar{\psi} \psi\rangle$}

Calculations of the lowest 8 eigenvalues for a set of MILC configurations have been used to evaluate the density of states at low virtuality, $\rho(0)$, and thereby numerically check the Banks-Casher relation on the lattice [23]. The results of low $\lambda$ fits to the integrated histograms yield values of $\langle\bar{\psi} \psi\rangle_{m_{q} \rightarrow 0}$ (to be compared with $\frac{\pi}{V} \rho(0)$ in parentheses) of $0.0114(0.0138)$ at $\beta=5.65$ and 0.002 $(0.002)$ at $\beta=5.725$.

The significant developments in the use of domain wall chiral fermions have resulted in a number of studies of zero modes reported at this conference [24]. For sufficiently large separation between domain walls, there is an integer index for the lattice Dirac operator corresponding to the winding number for smooth fields as well as exact zero modes [25]. In the presence of zero modes, the chiral condensate becomes $-\langle\bar{q} q\rangle=\frac{1}{V} \sum_{\lambda=0} \frac{1}{m}+\frac{1}{V} \sum_{\lambda>0} \frac{2 m}{\lambda^{2}+m^{2}}$, leading to a $\frac{1}{m}$ mass dependence. Recently [26], calculations with domain walls separated by 10 on a $16^{4}$ lattice in the presence of a smooth configuration with winding number one plus random fluctuations demonstrated this $\frac{1}{m}$ dependence over the range $10^{-5}<m<10^{-3}$.

\section{INSTANTON CONTENT OF THE QCD VACUUM}

Several recent studies of the instanton content of the $\mathrm{SU}(3)$ vacuum provide an unprecedented, albeit imperfect, view of the size distribution of instantons and their spatial correlations. To appreciate the issues involved in determining the instanton content, it is useful to briefly review the methods that have been used.

\subsection{Methods to extract the instanton content}

Standard cooling [15, 16] applied to QCD minimizes the action locally at each link. For the Wil- son action, one calculates $\operatorname{Max}_{[\mathrm{U}]}\left(\operatorname{Re} \operatorname{Tr} \mathrm{U} \Sigma^{\text {staples }}\right)$ so that $\mathrm{U}^{-1}$ is the projection on $\mathrm{SU}(\mathrm{N})$ of $\Sigma^{\text {staples }}=\sum_{\mu} \rightarrow$. In the case of $\mathrm{SU}(2)$, since the sum of staples is a multiple of a group element, cooling makes the replacement $\nmid \rightarrow \sum_{\mu} \rightarrow$. For $\mathrm{SU}(3)$, it is convenient to sequentially minimize with respect to $\mathrm{SU}(2)$ subgroups [27].

Underrelaxed cooling [28] takes a linear combination of the original link and the sum of staples, $\uparrow \rightarrow(1-c) \uparrow+\frac{c}{6} \sum_{\mu} \rightarrow 7$, which corresponds to APE smearing [29] and for small $c$ has the effect of making the minimization independent of the order of local updates. This has been used in Ref. [30] with $c=0.86$ and sequential application to $\mathrm{SU}(2)$ subgroups to analyze the UKQCD $\mathrm{SU}(3)$ configurations. This work also calibrates the cooling at each mixing parameter $c$ and $\beta$ to define a number of calibrated sweeps which produces approximately equivalent evolution of instantons and instanton-anti-instanton pairs. Instantons are identified by peaks in the topological charge density, $\mathrm{Q}(\mathrm{x})$, assuming additivity and filters are applied to assure the appropriate shape and to avoid counting fluctuations.

Renormalization group cycling, as originally implemented [31, is based on the renormalization group transformation $e^{S(U)}=$ $\int d[V] e^{S(V)+\kappa T(U, V)}$. The blocking transformation $\kappa T(U, V)$ maps a configuration $\mathrm{V}$ on a fine lattice to a configuration $\mathrm{U}$ on a lattice twice as course, and in the weak coupling limit the inverse transformation is given by the saddle point condition $\operatorname{Min}_{\{V\}}[S(V)+\kappa T(U, V)]$. Making use of the fact that $\mathrm{V}$ is the smoothest configuration that blocks to $\mathrm{U}$ and that the renormalization group preserves the instantons and large scale structure of the configuration, the cycling procedure first inverse blocks from the course lattice to the fine lattice and then reblocks back to a course sublattice shifted one lattice spacing along the diagonal.

Although cycling has many conceptual and practical advantages, including stabilizing instantons with $\rho>0.94 a$ and smoothing configurations sufficiently to identify instantons before the string tension has been severely diminished, it is very expensive computationally. Hence, efficient parameterizations of the cycling transfor- 
mation were studied [32] in SU(2). After investigating 56 combinations of paths up to 9 links, it was determined that a good parameterization was obtained by APE smearing, providing an additional rationale for this procedure. In addition, for reasons that are not yet clear, this work showed that although the sizes of smooth instanton configurations are stable under APE smearing, instantons and anti-instantons found in a configuration generated by the Wilson action may change size (generally growing) linearly with the number of steps. In Ref. [33], APE smearing $\uparrow \rightarrow\left[(1-c) \uparrow+\frac{c}{6} \sum_{\mu} \rightarrow\right]_{S U(3)}$ with direct projection onto $\mathrm{SU}(3)$ was used with $\mathrm{c}=0.45$ and 15-30 smearing steps to study the vacuum distribution of instantons. For comparison, UKQCD used $\mathrm{c}=0.86$ and 23-46 steps and the calculations of topological charge by the Pisa group discussed below used $\mathrm{c}=0.9$ and 2 steps.

Instead of sequentially minimizing the Wilson action, improved cooling [34] minimizes an improved action constructed from $\mathrm{n} \times \mathrm{m}$ plaquettes, $S=\sum_{\{n, m\}} c_{n, m} S_{n, m}$, where $S_{n, m} \equiv$ $\frac{1}{n^{2} m^{2}} \sum_{x, \mu, \nu} \operatorname{Tr}\left(1-m_{m} \eta\right)$. Up to five terms are included, corresponding to $1 \times 1,1 \times 2,1 \times 3,2 \times 2$, and $3 \times 3$ plaquettes, and the coefficients $c_{n, m}$ are determined to eliminate leading corrections in $a$ and also to create a slight barrier to prevent shrinkage of instantons above a critical size. Whereas the Wilson action for a smooth instanton monotonically decreases with $\rho$, leading to the eventual shrinkage and disappearance of instantons after hundreds of cooling steps, the five-term improved action is extremely flat and preserves all instantons greater than $\rho_{c}=2.3 \mathrm{a}$ indefinitely. A comparably improved topological charge operator yields nearly integer charge after 5-10 cooling steps. Improved cooling has also recently been used to study the $\mathrm{SU}(3)$ vacuum 35 .

A cooling method [20] that is particularly efficient for a parallel computer is simultaneous relaxation of the link variables at all lattice sites by discretizing the relaxation equation $\frac{d U}{d \tau}=-\frac{\delta S}{\delta U}$. Parameterizing an $\mathrm{SU}(3)$ matrix in the vicinity of $\mathrm{U}$ with by $U[F]=e^{i F} U$, where $\mathrm{H}$ is hermitian and traceless, and taking a small step $\Delta \tau$ yields $U^{\prime}=e^{-i \Delta \tau\left[\frac{1}{2} U U_{S}-\frac{1}{2}\left(U U_{S}\right)^{\dagger}-\frac{1}{N_{C}} \operatorname{Im} \operatorname{Tr}\left(U U_{S}\right)\right]} U$,
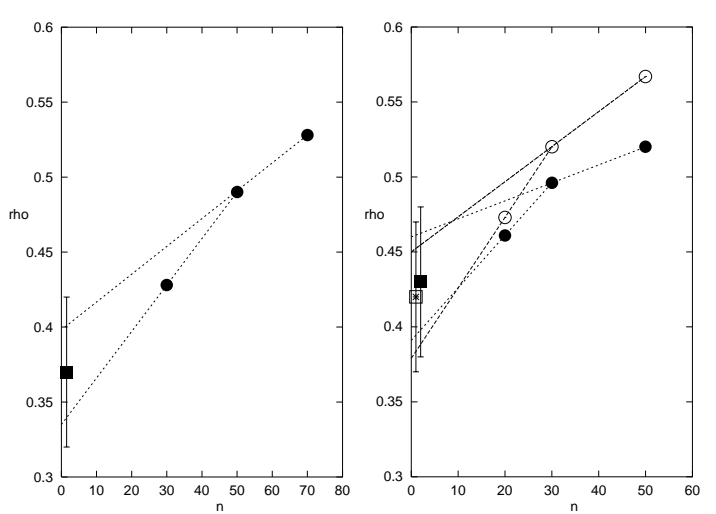

Figure 5. Sketch of extrapolations of the average instanton size to $\mathrm{n}_{c}=0$ cooling steps for data from Refs. 30] (left) and [20] (right). Solid and open symbols denote quenched and full QCD respectively.

where $U_{S}$ denotes the staple (or generalized staple for an improved action) associated with U. Sufficiently small $\Delta \tau$ produces cooling histories comparable to overrelaxed cooling, and $\Delta \tau=0.025$ was used to analyze $\mathrm{SU}(3)$ configurations.

\subsection{Vacuum instanton distribution}

Key features and salient results of five studies of instantons in the $\mathrm{SU}(3)$ vacuum are summarized in Table 1 .

A primary focus has been measuring the distribution of instanton sizes, $n(\rho)$, and the corresponding average size $\bar{\rho}$. The fundamental limitation is the fact that the distribution is modified to some extent by each cooling procedure. Although in principle, cooling with the Wilson action eventually allows instantons to shrink and eventually fall through the lattice, for the small number of cooling steps used with it in these studies, the effect is inconsequential. A serious problem for all methods, however, is the fact that sequential minimization of the action will rotate the relative color orientation of instanton-anti-instanton pairs into the attractive direction and bring them together until they annihilate. Even over the relatively small range of cooling steps in Ref. [30], for example, instanton number densities range from $55 \mathrm{fm}^{-4}$ to $3 \mathrm{fm}^{-4}$, and Refs. 17, 35. extend down to $0.3 \mathrm{fm}^{-4}$. Continued evolution would even- 
Table 1

Studies of the instanton content of the SU(3) Vacuum

\begin{tabular}{|c|c|c|c|c|c|c|}
\hline$\beta$ & Lattice & Method & $\begin{array}{l}\bar{\rho}_{\mathrm{N} / \mathrm{V}} \\
(\mathrm{fm})\end{array}$ & $\begin{array}{c}\bar{\rho}_{\text {extrap }} \\
(\mathrm{fm})\end{array}$ & $\begin{array}{c}\frac{N}{V} \\
\left(\mathrm{fm}^{-4}\right)\end{array}$ & Ref. \\
\hline $\begin{array}{l}6.2 \\
6.4\end{array}$ & $\begin{array}{l}16^{3} \times 48 \\
32^{3} \times 64 \\
24^{3} \times 48 \\
32^{3} \times 64\end{array}$ & $\begin{array}{l}\text { Underrelaxed } \\
\text { Cooling }\end{array}$ & $0.60(5)^{a}$ & $0.37(5)^{b}$ & $55-3.2$ & 30 \\
\hline $\begin{array}{l}5.85 \\
6.0 \\
6.1 \\
\end{array}$ & $\begin{array}{c}12^{4} \\
12^{4}, 16^{4} \\
16^{4}\end{array}$ & APE Smearing & & $0.32^{c}$ & 1.1 & 33] \\
\hline $\begin{array}{l}5.85 \\
6.0 \\
\end{array}$ & $\begin{array}{l}12^{4} \\
16^{4}\end{array}$ & $\begin{array}{l}\text { Improved } \\
\text { Cooling }\end{array}$ & $<0.53(5)^{d}$ & & $3.3-0.38$ & 35 \\
\hline 5.7 & $16^{3} \times 24$ & Cooling & & $>0.39^{e}$ & $0.59-0.28^{f}$ & 17 \\
\hline 5.85 & $16^{4}$ & Relaxation & $0.50(5)^{g}$ & $0.43(5)^{b, g}$ & $5.3-1.4^{g}$ & 20 \\
\hline \multicolumn{2}{|c|}{ unquenched, $\kappa=0.1$} & & $0.52(5)^{g}$ & $0.42(5)^{b, g}$ & $6.5-1.8^{g}$ & \\
\hline \multicolumn{3}{|c|}{ Summary } & $0.54(5)$ & $0.39(5)$ & & \\
\hline
\end{tabular}

${ }^{a}$ Value $0.56(5)$ at $N / V=8.5$ for $\beta=6.4$ evolved to $N / V=3.2$ using $\beta=6.0$ data.

${ }^{b}$ Extrapolation sketched in Fig. 5

${ }^{c}$ Value 0.3 scaled $5.6 \%$ using $a(\sqrt{\sigma}=440 \mathrm{MeV})$.

${ }^{d}$ From graphs of $N / V=1.81$ and 1.43 data. Evolution to 3.2 would reduce $\rho$ further.

$e$ Value 0.36 scaled $9 \%$ using $a(\sqrt{\sigma}=440 \mathrm{MeV})$. Correlation function range underestimates average $\rho$ 34.

${ }^{f}$ Lattice spacing increased $9 \%$ using $a(\sqrt{\sigma}=440 \mathrm{MeV})$.

$g$ Lattice spacing from hadron masses increased $18 \%$ using $a(\sqrt{\sigma}=440 \mathrm{MeV})$.

tually annihilate all the pairs until only instantons or anti-instantons remain. The trade-off in all measurements is the uncertainty in identifying instantons and anti-instantons in the presence of fluctuations for a small number of steps versus the damage to the original instanton ensemble caused by a large number of steps.

To compare the various calculations and emphasize the similarity of their essential features as much as possible, two alternative definitions of the average instanton size have been used in Table 1. One definition is the density extrapolated in the number of cooling steps, $n_{c}$, to $n_{c}=0$. This is more representative of the original, unfiltered vacuum but more subject to pattern recognition errors. As mentioned previously, it is necessary for APE smearing where instanton sizes were observed to evolve linearly. Data from Refs. 20, 30 are plotted in Fig. 5 with linear extrapolations and the error bars bracketing these extrapolations are listed in Table 1. The calculation in Ref. 17 is effectively an extrapolation, since the scale calculated from hadron masses evolves nearly linearly with the number of cooling steps.

The second definition is the continuum limit of comparably filtered configurations. Ref. 30 defines a calibrated number of cooling sweeps $n_{c}(\beta)$ to reach the same density of instantons $\frac{N}{V}$ at each $\beta$ and extrapolates linearly in $a^{2}$ to the continuum limit. This is consistent with the fact that observables such as the instanton distribution shown in Fig. 6 agree well at $\beta=6.0,6.2$, and 6.4.

To facilitate comparison in Table 1, as indicated in the footnotes, authors' data were scaled to a lattice spacing determined by the string tension $\sqrt{\sigma}=440 \mathrm{MeV}$ and $\bar{\rho}_{\frac{N}{V}}$ was evaluated as close to $\frac{N}{V}=3.2 \mathrm{fm}^{-4}$ as possible. From the table, there is qualitative agreement among all the calculations and the extrapolated value $\bar{\rho}_{\text {extrap }}$ lies in the range $0.39 \pm .05 \mathrm{fm}$, consistent with the in- 

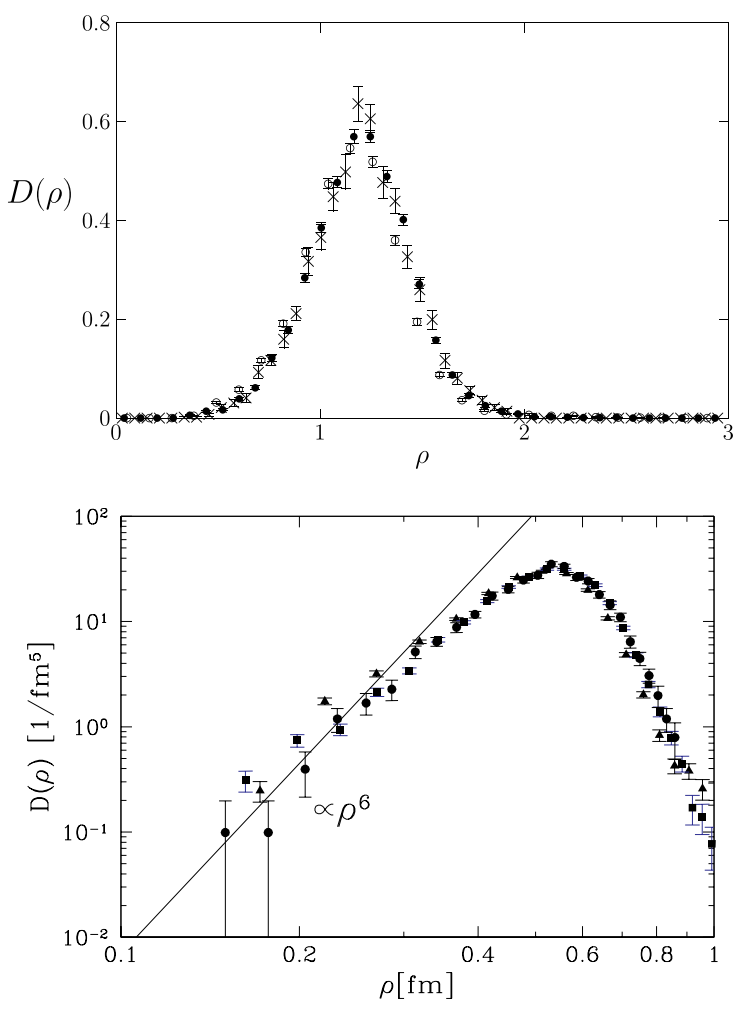

Figure 6. UKQCD instanton size distribution 30. In top plot, $\rho=1$ corresponds to $0.45 \mathrm{fm}$.

stanton liquid model, while the value after cooling to $\frac{N}{V}$ on the order of $3.2 \mathrm{~m}^{-4}$ yields $\bar{\rho}_{\frac{N}{V}}$ substantially larger, in the range $0.54 \pm .05 \mathrm{fm}$. Although no attempt has been made to calculate $\frac{N}{V}$ characterizing the uncooled vacuum, it is clear that the density of instantons identified during the early stages of cooling is substantially larger than the value $1 \mathrm{fm}^{-4}$ in the instanton liquid model, which should likely be thought of as an effective density in the size range dominating physical processes.

Each of the studies in Table 1 also calculated the distribution of instanton sizes, with similar results. The UKQCD results [30], which had the highest statistics and greatest range of parameters, are shown in Fig. 6. The upper plot shows the high level of consistency obtained between comparably cooled configurations with $\beta$ $=6.0,6.2$, and 6.4 for 23,46 , and 80 cooling steps denoted by open circles, closed circles, and

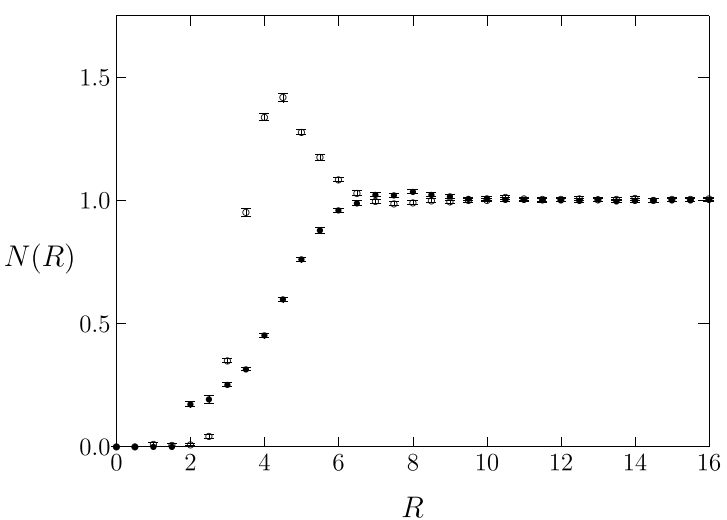

Figure 7. Distribution of like (solid circles) and opposite (open circles) charges as a function of distance $\mathrm{R}$ from a reference charge [30], where $1 \mathrm{fm}$ corresponds to $\mathrm{R}=2.2$

crosses. Similar consistency was demonstrated at $\beta=6.0$ between configurations on $16^{3} \times 48$ and $32^{3} \times 64$ lattices. Full QCD with $\frac{m_{\pi}}{m_{\rho}}=0.82$, has a similar distribution [20]. The log-log plot [36] of the UKQCD data shows that the distribution of instantons grows roughly as $\rho^{6}$ at small $\rho$ as expected in the dilute approximation, falls significantly below $\rho^{6}$ in the region of $0.3 \mathrm{fm}$, and decreases at large $\rho$ much more rapidly than $\rho^{5}$ characterizing a frozen coupling constant.

Interesting correlations between instantons have also been observed [30]. Fig. 7 shows the distribution of like and unlike topological charges as a function of the distance from a reference charge and clearly reflects the effect of instanton-antiinstanton attraction. Quantitatively, the average distances to the nearest like and unlike charges are 0.49 and $0.45 \mathrm{fm}$ respectively, to be compared with the average size $0.56 \mathrm{fm}$. In a dilute gas, the distribution would be Poisson implying $\left\langle Q^{2}\right\rangle=N_{I}+N_{A}$, which only holds for $\rho<0.5 \mathrm{fm}$. By all measures, the instanton distribution is not dilute, and is best thought of as a liquid. In addition, smaller and larger instantons tend to have opposite charge, with the smaller instantons having the sign of the total $\mathrm{Q}$, so that the large charges are overscreened. Many other interesting details are found in 30. 
Table 2

Topological Susceptibility

\begin{tabular}{|c|c|c|}
\hline$\chi^{1 / 4}(\mathrm{MeV}$ & Method & Ref. \\
\hline \multicolumn{3}{|c|}{$\mathrm{SU}(2)$} \\
\hline $230(30)$ & RG Cycling & 31 \\
\hline $220(6)$ & APE Smearing & 32 \\
\hline $200(15)$ & Improved Cooling & 34] \\
\hline $198(8)$ & $\mathrm{APE}+$ Renorm. & \\
\hline $226(4)$ & $\begin{array}{l}\text { Geometric + Renorm. } \\
\text { Spectral Flow }\end{array}$ & 38 \\
\hline \multicolumn{3}{|c|}{$\mathrm{SU}(3)$} \\
\hline $187(14)$ & Underrelaxed Cooling & 30 \\
\hline $192(5)$ & APE Smearing & 33 \\
\hline $185(9)$ & Improved Cooling & 35 \\
\hline $175(5)$ & APE + Renorm. & [40] \\
\hline $197(4)$ & Spectral Flow & 39 \\
\hline 180 & Veneziano-Witten & 7,8 \\
\hline
\end{tabular}

\subsection{Topological susceptibility}

It appears that we now understand how to define quantities on a lattice that correspond to the topological charge in the continuum limit and that, as shown in Table 2, all methods for calculating the topological susceptibility are reasonably consistent with each other and with the Veneziano-Witten formula.

As emphasized in Ref. 38, all measurements of the susceptibility involve both additive and multiplicative renormalization, $a^{4} \chi^{i}=$ $Z(\beta)^{-2}\left(\chi_{\text {lattice }}^{i}-M(\beta)\right)$. By placing known smooth configurations on a lattice and heating by Monte Carlo updates, $\mathrm{M}$ was measured for Lüscher's geometrical charge and both $\mathrm{M}$ and $\mathrm{Z}$ were measured for the clover approximation to $\mathrm{F} \tilde{\mathrm{F}}$ with zero and two APE smearing steps, leading to a consistent value of $\chi$ for all three calculations [38]. Cooling automatically removes the additive term and brings $\mathrm{Z}$ to unity by removing quantum fluctuations. It determines the susceptibility accurately because the total charge is unaffected by pair annihilation and because, with improved charge operators, the charge converges to a stable integer after very few cooling steps. Hence, the various cooling methods yield consis- tent results as shown. Finally, determination of the topological charge from spectral flow [39] also appears to be consistent with other methods.

The susceptibility in SU(3) has also been studied using the geometrical charge [37]. The Wilson action yields $\chi^{\frac{1}{4}}=228 \mathrm{MeV}$, which is expected to be high because of the additive contribution from dislocations. Calculations with a renormalization group improved action that suppresses dislocations appear to have less additive renormalization, but lack of an accurate measurement of the scale precludes quantitative comparison.

\subsection{Equilibration of topological sectors}

An important problem in Monte Carlo calculations with dynamical fermions is ergodically sampling the relevant topological sectors. Recent hybrid Monte Carlo calculations 41 with 3000 to 5000 trajectories on $16^{3} \times 32$ lattices using Wilson fermions have demonstrated sufficient tunneling to achieve equilibration of $\mathrm{Q}$ at $\frac{m_{\pi}}{m_{\rho}}=0.84,0.76$, and 0.69. At the lightest quark mass, an approximately Gaussian distribution of $\mathrm{Q}$ is observed and the integrated autocorrelation time for $\mathrm{Q}, \tau=$ $54(4)$, is only moderately larger than the value $\tau=42$ (4) for other observables. For $\frac{m_{\pi}}{m_{\rho}}=.56$, the mean value of $\mathrm{Q}$ did not equilibrate to 0 within 3,500 trajectories. These results are consistent with previous HMC calculations with staggered fermions, for which autocorrelation times of the order of 10 were observed for $\frac{m_{\pi}}{m_{\rho}}=0.75$ and 0.65 , but $\frac{m_{\pi}}{m_{\rho}}=0.57$ exhibited little mobility over 450 trajectories [42]. Thus, there is an intermediate range of masses down to $\frac{m_{\pi}}{m_{\rho}} \sim 0.69$ for which equilibration of topological sectors is achieved for both Wilson and staggered fermions.

\section{INSTANTONS AND THE STATIC POTENTIAL}

Several puzzles concerning the contribution of instantons to the static potential have emerged in recent years. A provocative calculation 443. suggested that an instanton liquid with a distribution $\sim \rho^{-5}$ at large $\rho$ produced a linear confining potential with the physical string tension. In addition, a confining potential appears to survive renormalization group cycling for $\mathrm{SU}(2)$, but 
largely disappear when the instantons identified in the cycled configuration are replaced by instantons of comparable size and random orientation [31].

These and related issues are addressed by high statistics calculations 44 of the potentials in an instanton liquid with fixed instanton size and with the size distribution $\rho^{6}\left(\rho_{0}^{3.5}+\rho^{3.5}\right)^{-\frac{11}{3.5}}$, corresponding to a frozen coupling constant . The potentials are linear at small distance and approach a constant at large distance as expected analytically [45]. With $\bar{\rho}=\frac{1}{3} \mathrm{fm}$ and $\frac{N}{V}=1 \mathrm{fm}^{-4}$, both distributions yield the same slope, $\sigma=0.11$ $\mathrm{GeV} / \mathrm{fm}$, roughly one tenth of the physical string tension. Whereas the fixed size potential approaches a constant already by $1.5 \mathrm{fm}$, the $\rho^{-5}$ potential is still rising substantially at $3 \mathrm{fm}$ due to the contributions of large instantons. Because the potential is roughly proportional to $\frac{N}{V}$ and since by scaling, the physical string tension would be obtained at $\frac{N}{V}=1 \mathrm{fm}^{-4}$ by increasing $\bar{\rho}$ by $10^{\frac{1}{4}}$ to $0.59 \mathrm{fm}$, one can understand several other results. The peak of the distribution in Ref. 43] is at $0.4 \mathrm{fm}$ corresponding to $\bar{\rho}$ above $0.5 \mathrm{fm}$, so the slope should be near the physical value. Also, the potential only extended to $1 \mathrm{fm}$, so the departure from confinement was not observed. Similarly, cooled distributions with larger instanton density and $\bar{\rho}$ are also enhanced.

The question of what correlations remain in the renormalization group cycled configurations yielding either confinement or at least a large slope out to $1 \mathrm{fm}$ that are not contained in a comparable random distribution of instantons is interesting and should be pursued. One suggestive feature is the fact that an instanton liquid as usually constructed does not have $\mathrm{Z}(\mathrm{N})$ symmetry, whereas confining configurations generated by sampling the action automatically have it.

\section{INSTANTONS AT NONZERO TEMPERATURE}

\subsection{Topological susceptibility}

Measurements of the topological susceptibility beyond the critical temperature confirm the idea that instantons should be suppressed by Debye screening, where the leading suppression fac- tor [46] is $e^{-\frac{1}{3}\left(2 N_{c}+N_{f}\right)(\pi \rho T)^{2}}$ As at zero temperature, within one's ability to read numbers from graphs, quenched measurements using one and two APE smearing steps corrected for additive and multiplicative renormalization 40] agree with analysis of cooled configurations [17]. The cooled measurements showed that the average instanton size decreased from $\bar{\rho}=0.33 \mathrm{fm}$ at low temperature to $0.26 \mathrm{fm}$ at $1.34 \mathrm{~T}_{C}$ and that with the latter value, Debye screening fit the rapid fall off of the susceptibility. Comparable cooling calculations with staggered fermions [48] showed analogous results, with the instanton size $\bar{\rho}=0.44 \mathrm{fm}$ at $.75 \mathrm{~T}_{C}$ decreasing to $0.33 \mathrm{fm}$ at $1.3 \mathrm{~T}_{C}$ and Debye screening with the latter size fitting the susceptibility above $\mathrm{T}_{C}$. The transition is considerably sharper than in the unquenched case, consistent with the $\mathrm{N}_{f}$ dependence of the Debye screening.

\subsection{Calorons}

Gauge fields at nonzero temperature may be classified by the Polyakov loop $\mathcal{P}_{\infty}=$ $\operatorname{Lim}_{x \rightarrow \infty} P e^{\int_{0}^{\beta} d t A_{0}(\vec{x}, t)}$, and one usually considers $\mathcal{P}_{\infty}= \pm 1$ since fluctuations around classical solutions with other values produce a non-zero vacuum energy density [49]. The periodic instanton, or caloron is given by $A_{\mu}(x)=\frac{i}{2} \bar{\eta}_{\mu \nu}^{3} \tau_{3} \partial_{\nu} \ln \phi$, with $\phi$ a periodic sum of instantons displaced in the thermal direction [50]. In the limit where the instanton size $\rho$ is large compared with $\beta$, the spatial distribution becomes independent of $t$, and gauge transformation yields a BPS monopole at the center of the instanton (and a monopole with opposite charge at infinity [51].

During the past year, a remarkable set of solutions comprised of monopoles has been discovered 52.53 for nontrivial values of $\mathcal{P}_{\infty}$, corresponding to to a periodic array of instantons twisted by $\mathcal{P}_{\infty}$. The closed form solutions are surprisingly simple. For $\mathrm{SU}(2)$ with $\mathcal{P}_{\infty}=e^{2 \pi i \omega \tau_{3}}$ the action density is $\operatorname{tr} F_{\mu \nu}^{2}=\partial_{\mu}^{2} \partial_{\nu}^{2} \ln \psi$, where $\psi=$ $-\cos (2 \pi t)+c_{1} c_{2}+\frac{r_{1}^{2}+r_{2}^{2}+\pi^{2} \rho^{2}}{2 r_{1} r_{2}} s_{1} s_{2}+\pi \rho^{2}\left(\frac{s_{1} c_{2}}{r_{1}}+\frac{s_{2} c_{1}}{r_{2}}\right)$, the positions of two constituent monopoles are denoted $r_{m}=\left|\vec{x}-\vec{y}_{m}\right|$ for $\mathrm{m}=1,2,\left|\vec{y}_{2}-\vec{y}_{1}\right|=\pi \rho^{2}$, $\nu_{1}=2 \omega, \nu_{2}=1-2 \omega, c_{m}=\cosh \left(2 \pi \nu_{m} r_{m}\right)$, $s_{m}=\sinh \left(2 \pi \nu_{m} r_{m}\right)$, and the solution is scaled 


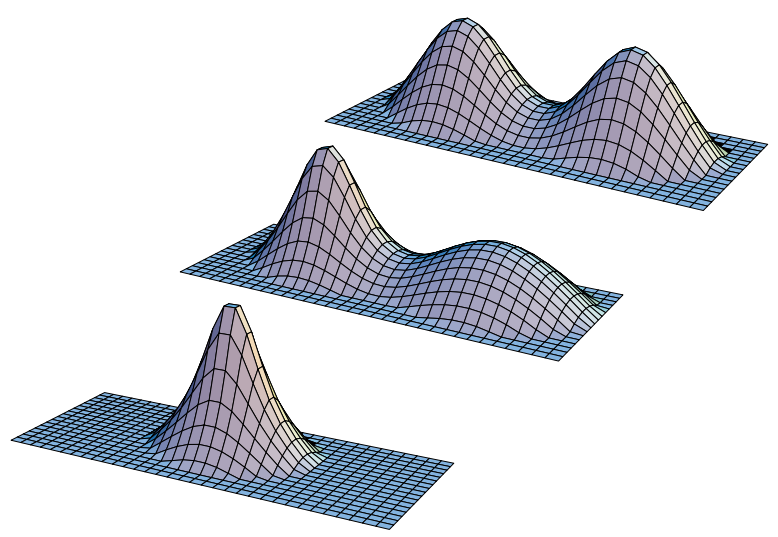

Figure 8. Action density profiles for calorons $[52]$ at $\omega=0, \frac{1}{8}$, and $\frac{1}{4}$ with $\rho=\beta=1$.

to $\beta=1$. General expressions are given for $\operatorname{tr} F_{\mu \nu}^{2}$ and $A_{\mu}(x)$ for SU(N) in Ref. [52].

In the $\mathrm{SU}(2)$ case, for $\rho$ large relative to $\beta$ the the solution separates into two lumps separated by $\frac{\pi \rho^{2}}{\beta}$ with monopole charges \pm 1 , masses $\frac{8 \pi^{2} \nu_{m}}{\beta}$, and widths $\sim \frac{1}{\nu_{m}}$. In the limit of large $\frac{\rho}{\beta}$ the lumps become well separated, spherically symmetric, and time independent. Since they are selfdual, they are therefore BPS monopoles. As $\omega$ approaches 0 or $\frac{1}{2}$, the second lump becomes negligible and the solution becomes the spherically symmetric Harrington-Shepard caloron. The action densities plotted on logarithmic scales in Fig 8 at $\mathrm{t}=0$ show the separation into lumps at $\rho=\beta$. For small $\frac{\rho}{\beta}$, the caloron approaches a single instanton solution. In the maximal abelian gauge, as $\rho$ increases from 0 to $\infty$, the small monopole loops associated with instantons grow. When these loops extend from 0 to $\beta$, they merge with their periodic extensions to produce pairs of monopole lines which eventually become the well-separated BPS monopoles [54]. In the case of $\mathrm{SU}(\mathrm{N})$, the solution generalizes to $\mathrm{N}$ monopole lumps, and results are shown in Ref. [52].

These novel finite temperature solutions provide interesting examples of gauge fields with topological charge which are built out of monopoles without gauge fixing, and we may hope that they will lead us a step closer to analytical insight into the role of monopoles in QCD.

\section{SUMMARY AND OPEN PROBLEMS}

We have come a long way in exploring instanton physics on the lattice. The behavior of twopoint functions, the similarity of cooled and uncooled calculations, and the contributions of zero modes provide substantial evidence that instantons play a major role in the physics of light hadrons. There has been significant progress in calculating quark zero modes, observing localization, extracting the instanton content of the vacuum, calculating the topological susceptibility, and discovering analytic solutions with monopole constituents of instantons. Salient problems for further investigation include reducing the ambiguity in the instanton distribution in the vacuum, removing the effect of doublers in spectral flow analyses, understanding the role of instantons in confinement, and especially studying full QCD with light chiral fermions, where the contributions of instantons and zero modes become most significant.

\section{Acknowledgments}

It is a pleasure to acknowledge Richard Brower, Dong Chen, Ming Chu, Philippe de Forcrand, Tom DeGrand, Anna Hasenfratz, Suzhou Huang, Taras Ivanenko, Andrew Pochinsky, Edward Shuryak, and Uwe-Jens Wiese for enlightening discussions and insights.

\section{REFERENCES}

1. A.A. Belavin, A.M. Polyakov, A.P. Schwartz, and Y.S. Tyupkin, Phys. Lett. B 59 (1975) 85.

2. C.G. Callan, R. Dashen, and D. J. Gross, Phys. Rev. D 17 (1978) 2717.

3. E.V. Shuryak Nucl. Phys. B 198 (1982) 83.

4. D.I. Diakanov and V.Y. Petrov, Nucl. Phys. B 245 (1984) 259; 272 (1986) 457.

5. T. Schäffer and E.V. Shuryak, Rev.Mod.Phys. 70 (1998) 323, and references therein.

6. R. Narayanan, talk at this conference and references therein.

7. E. Witten, Nucl. Phys. B 156 (1979) 269.

8. G. Veneziano, Nucl. Phys. B 159 (1979) 213.

9. T. Banks and A. Casher, Nucl. Phys. B 169 (1980) 103.

10. E.V. Shuryak, Rev. Mod. Phys. 65 (1993) 1. 
11. M.-C. Chu, J.M. Grandy, S. Huang, and J.W. Negele, Phys. Rev. D 48 (1993) 3340.

12. E.V. Shuryak and J.J.M. Verbaarschot, Nucl. Phys. B 410 (1993) 55.

13. T. Schäfer, E.V. Shuryak, and J.J.M. Verbaarschot, Nucl. Phys. B 412 (1994) 143.

14. S.J. Hands, P.W. Stephenson, A. McKerrell, Phys. Rev. D 51 (1995) 6394.

15. B. Berg, Phys. Lett. B 104 (1981) 475.

16. M. Teper, Nucl.Phys.B(Proc.Suppl.)20(1991) 159.

17. M.-C. Chu, J.M. Grandy, S. Huang, and J.W. Negele, Phys. Rev. D 49 (1994) 6039.

18. M.-C. Chu, M.Lissia, and J.W. Negele, Nucl. Phys. B 360 (1991) 31; M. Lissia, M.-C. Chu, J.W. Negele, and J.M. Grandy, Nucl. Phys. A 555 (1993) 272.

19. D. Dolgov, R.C. Brower, J.W. Negele, and A. Pochinhsky, hep-lat/9809132, talk at this conference.

20. T.L. Ivanenko and J.W. Negele, Nucl. Phys. B (Proc.Suppl.) 63 (1998) 504; T.L. Ivanenko, MIT Ph.D. thesis, 1997; J.W. Negele, heplat/9804017.

21. L. Venkataraman and G. Kilcup, heplat/9711006.

22. C. Gattringer and I. Hip, hep-lat/9712015v2, hep-lat/9806032.

23. J.E. Hetrick, talk at this conference.

24. T. Blum, talk at this conference.

25. R. Narayanan and H. Neuberger, Phys.Lett.B 302 (1993) 62; Phys. Rev. Lett. 71 (1993) 3251;

Nucl. Phys. B 412 (1994) 574; ibid. 443 (1995) 305.

26. P. Chen, N. Christ, G. Fleming, A. Kaehler, C. Malureanu, R. Mawhinney, C. Sui, P. Vranas, and Y. Zhestkov, hep-lat/9807029.

27. N. Cabibbo and E. Marinari, Phys. Lett. B119 (1982) 387 .

28. C. Michael and P. Spencer, Phys. Rev. D 52 (95) 4691.

29. M. Falcioni, M. Paciello, G. Parisi, B. Taglienti, Nucl. Phys. B 251[FS13] (1985) 624; M. Albanese et. al., Phys. Lett. B 192 (1987) 163.

30. D.A. Smith and M.J. Teper, hep-lat/9801008.

31. T. DeGrand A. Hasenfratz, and T.G. Kovács, Nucl. Phys. B 505 (1997) 417.

32. T. DeGrand A. Hasenfratz, and T.G. Kovács,
Nucl. Phys. B 520 (1998) 301.

33. A. Hasenfratz and C. Nieter, hep-lat/9807002.

34. Ph. de Forcrand, M. García Pérez, and I.O. Stamatescu, Nucl. Phys. B (Proc.Suppl.) 47 (1996) 777; Nucl. Phys. B 499 (1997) 409.

35. Ph. de Forcrand, M. García Pérez, G. Münster and I.-O.Stamatescu, SCSC-TR-98-12; Ph. de Forcrand, M. García Pérez, J.E. Hetrick, and I.-O. Stamatescu, hep-lat/9802017.

36. A. Ringwald and F. Schrempp, hep-ph/9805492.

37. J. Grandy and R. Gupta, Nucl. Phys. B (Proc.Suppl.) 34 (1994) 164; ibid42 (1995) 246; J. Grandy and G. Kilcup, ibid 53 (1997) 560.

38. B. Allés, M. D'Elia, A. Di Giacomo, and R. Kerchner, hep-lat/9711026v2.

39. R. Edwards, talk at this conference

40. B. Allés, M. D'Elia, and A. Di Giacomo, Nucl. Phys. B 494 (1997) 281.

41. B. Allés et. al.,hep-lat/9803008.

42. B. Allés G. Boyd, M. D'Elia, A. Di Giacomo, and E. Vicari, Phys. Lett. B 389 (1996) 107.

43. M. Fukushima, H.Suganuma, A. Tanaka, H. Toki, S Sasaki, Nucl. Phys. B (Proc.Suppl.) 63 (1998) 513.

44. R.C. Brower, D. Chen, J.W. Negele, and E.V. Shuryak, hep-lat/9809091.

45. C. Callan, R. Dashen, D. Gross, F. Wilczek, and A. Zee, Phys. Rev. D 18 (1978) 4684.

46. R.D. Pisarski and L.G. Yaffe, Phys. Lett. B 97 (1980) 110.

47. M.-C. Chu and S. Schramm, Phys. Rev. D 51 (1995) 4580.

48. M.-C. Chu, S.M. Ouellette, S. Schramm, and R. Seki, hep-lat/9712023.

49. D.J. Gross, R.D. Pisarski, and L.G. Yaffe, Rev. Mod. Phys. 53 (1981) 43.

50. B.J. Harrington and H.K. Shepard, Phys. Rev. D 17 (1978) 2122; 18 (1978) 2990.

51. P. Rossi, Nucl. Phys. B 149 (1979) 170.

52. T.C. Kraan and P. van Baal, Phys. Lett. B 428 (1998) 268; hep-th/9805168; hep-th/9805201; hep-th/9806034; hep-lat/9808015.

53. K. Lee, Phys. Lett. B 426 (1998) 323; K. Lee and C. Lu, Phys. Rev. D 58 (1998) 025011.

54. R.C. Brower, K. Chen, J.W. Negele, K. Orginos, and C.-I. Tan, hep-lat/9810009. 